\title{
Self-control in honeybees
}

\author{
KEN CHENG, JENNIFER PEÑA, MELANIE A. PORTER, and JULIA D. IRWIN \\ Macquarie University, Sydney, Australia
}

\begin{abstract}
Self-control means choosing a large delayed reward over a small immediate reward; impulsiveness is its opposite. The metabolic hypothesis states that the amount of self-control across species correlates negatively with metabolic rate (Tobin \& Logue, 1994). Foraging honeybees have high metabolic rates; the metabolic hypothesis would predict little self-control in bees. But foraging bees work for the longterm good of their hive, conditions that seem to require self-control. In three experiments, we gave bees the choice between (1) a sweeter delayed reward and a less sweet immediate reward and (2) a large delayed reward and a small immediate reward. Bees showed much self-control, inconsistent with the metabolic hypothesis.
\end{abstract}

Self-control means choosing a large delayed reward over a small immediate reward; its opposite is impulsiveness. A lack of self-control is central in many contemporary human problems, such as overeating (Forzano \& Corry, 1998), drug abuse, failures at school, poor money management, lack of social ability, depression, and criminal tendencies (see Baumeister, Heatherton, \& Tice, 1994, and Logue, 1988,1998 , for reviews). These problems may have evolutionary roots. Logue $(1988,1995,1998)$ argued that in the evolutionary past of humans, the future was far more uncertain than it is now and it may have paid to "grab what we can." When one is faced with uncertain environments and doubtful futures, waiting for a larger reinforcer (which may never arrive) could be unwise, and impulsiveness might be the best strategy (Green, Fry, \& Myerson, 1994; Logue, 1988; Tobin, Logue, Chelonis, Ackerman, \& May, 1996). Self-control, on the other hand, would be adaptive when an immediate reinforcer is unnecessary and when the environment is constant enough that the availability of reinforcers is quite certain (Logue, 1988). In today's more predictable world, evolution may have supplied us with less self-control than is optimal.

Much research on self-control has been conducted on vertebrate animals in laboratory settings. Pigeons have consistently shown a preference for immediate small rewards over large delayed rewards with keypecking (e.g., Ainslie, 1974; Green, Fisher, Perlow, \& Sherman, 1981; Green, Price, \& Hamburger, 1995; Grosh \& Neuringer, 1981; Mazur \& Logue, 1978) or treadle pressing (Chelonis \& Logue, 1996) as responses. Rats also typically show impulsive behavior in an operant chamber (Green et al., 1981; Logue, Rodriguez, Peña-Correal, \& Mauro, 1984; Tobin, Chelo-

The research reported was supported by grants from Macquarie University. We thank Dagong Zhang and Laura Anthony for help in gathering data and Mark Peterson for hive maintenance. Correspondence concerning this article should be sent to K. Cheng, Department of Psychology, Macquarie University, Sydney NSW 2109, Australia (e-mail: kcheng@ axon.bhs.mq.edu.au). nis, \& Logue, 1993) or a two-lane runway (Boeheme, Blakely, \& Poling, 1986).

Our experiments are concerned with the functional reasons for self-control. At least two different functional hypotheses have been proposed. One hypothesis states that metabolic rates determine self-control (Tobin \& Logue, 1994). Tobin and Logue examined a number of studies from Logue's laboratory on pigeons, rats, and humans. The amount of self-control was measured as a deviation from the prediction based on the matching law. The matching law states that

$$
\frac{B_{\mathrm{sc}}}{B_{\mathrm{imp}}+B_{\mathrm{sc}}}=\frac{A_{\mathrm{sc}} D_{\mathrm{imp}}}{A_{\mathrm{imp}} D_{\mathrm{sc}}+A_{\mathrm{sc}} D_{\mathrm{imp}}},
$$

where $B$ stands for response choices, $A$ stands for amount, and $D$ stands for delay. The subscript "sc" indicates the choice representing self-control, whereas "imp" indicates parameters for the impulsive choice. This measure was used to make comparisons across paradigms and species, with different parameters of delay and amount (Tobin \& Logue, 1994). Adult humans showed the most self-control, rats showed far less, and pigeons showed the least amount of self-control of all three. Examining the specific metabolic rates of each species, the authors found that the amount of self-control was negatively correlated with specific metabolic rate. A linear regression predicted the crossspecies data $(r=-.97)$. Tobin and Logue argued that animals with fast metabolisms need to be impulsive, since they require frequent feedings to survive.

Logue's $(1988,1995,1998)$ ecological analysis can be considered a second hypothesis. Its central theme of relating ecological conditions of life to the degree of selfcontrol exhibited by a species makes eminent sense. Unfortunately, however, it is at present too vague to make precise cross-species comparisons. It is not clear, for example, how the hypothesis would rank humans, rats, pigeons, and bees, species with which we will be concerned.

The eusocial honeybee gives an excellent test of the metabolic hypothesis. The beehive contains one queen, whose 
sole job is to reproduce eggs. The vast majority of the hive are workers, sterile females who do all the work necessary to keep the hive functioning. Workers do all the jobs, inside and outside their hive, at different stages of their lives, a pattern known as age polytheism. The honeybee has been tested in many experiments on learning (for a review, see Bitterman, 1996), and much about its navigational abilities is known (for reviews, see Cheng, 2000, and Collett \& Zeil, 1998). The socioecology and decision processes within the hive are also well studied (Seeley, 1985, 1995). We have failed, however, to find any studies on bees that pitted a larger delayed reward against a smaller immediate reward.

The bee is far smaller than rats and pigeons, offering a new, as yet untested size, and it is distant phylogenetically. Its specific metabolic rate is some four times that of pigeons (Hepburn, 1986; Seeley, 1985). By the metabolic hypothesis, the honeybee should show little self-control.

An ecological analysis, however, seems to make the opposite prediction. Logue (1988) claims that "self-control would be adaptive when immediate food intake is unnecessary and long-term survival is of paramount importance" (p. 678). Workers of eusocial insects such as honeybees typically work for the good of their nest. Returning foragers usually unload their food to food storers in the hive and then go off foraging again (Seeley, 1995). Being infertile, the worker's gene pool lies in her nest. For the propagation of her genes, the well-being of the nest is of utmost importance. By this ecological analysis, foraging honeybees in our experimental paradigms might be expected to show a good deal of self-control, at least more than rats in laboratory tests. We thus examined self-control in honeybees in three experiments. Experiment 1 pitted a sweeter delayed reward against a less sweet immediate reward, whereas Experiments 2 and 3 pitted a large delayed reward against a small immediate reward, both of the same quality.

\section{EXPERIMENT 1}

\section{Method}

\section{Subjects}

Honeybees $(n=16)$ lived in a nearby maintained hive. They were recruited to the laboratory with a reward of intermediate strength (2:3-parts sugar:water) placed in a bottle cap $(2.5 \mathrm{~cm}$ in diameter). Bees that returned to the gangplank ( $2 \mathrm{~cm}$ wide) leading into the laboratory (through a $2-\mathrm{cm}$ square opening) were painted for individual identification.

\section{Setup}

The bees' task was to walk through the entrance and fly to a table (120 cm wide, $75 \mathrm{~cm}$ deep, $73 \mathrm{~cm}$ tall). On the table were a sweeter (1:1-part sugar:water) solution, covered by a transparent glass, and a less sweet (1:4-parts sugar:water) solution, which was not covered. The two solutions in bottle caps were $62.4 \mathrm{~cm}$ apart. For half the bees, the sweeter reward was on the right side, whereas for the other half, it was on the left side. Directly between the bottle caps was a landmark, a cylinder covered by yellow poster paper $(9 \mathrm{~cm}$ diameter, $9 \mathrm{~cm}$ high). Under each bottle cap was a 10-cm-square piece of poster paper, half light blue and half yellow. The blue half was closer to the landmark on each side. The poster papers were switched frequently between the two sides to render any odors irrelevant. The landmark and poster papers were attempts to differentiate the two choices for the bees.

\section{Procedure}

Two bees participated on each day, but only 1 bee was allowed to enter the laboratory at a time, in order to make choices independent. A guillotine style door allowed gate control. Each bee was first trained to fly to a reward (2:3-parts sugar:water) at the center of the table, in front of the landmark. Then she was forced to sample each choice 20 times, in alternation. On such forced choices, only one reward was set up on the table. For the sweeter reward, the glass was removed by hand $5 \mathrm{sec}$ after the bee entered the area delimited by the poster paper under the cap. After the forced choices, both choices were available. We recorded which cap the bee drank from. It was possible for the bee to leave the sweeter delayed reward during the course of the delay. Once a bee started drinking, it always drank to repletion. It was then released to fly home and returned of its own accord (typically, in 5-10 $\mathrm{min}$ ). The bees were given 20-34 choice tests.

For bees that did not choose the delayed sweeter reward at greater than chance levels ( $p<.05$ by the binomial test), we tested whether they preferred the sweeter solution when its access did not have a delay. Each bee was forced to sample each solution (now both with no delay) 10 times and then was given choice tests (15-41).

\section{Results and Discussion}

The proportion of choices of the sweeter delayed reward by individual bees is shown in Figure 1. Also shown in Figure 1 are the proportions of sweeter reward chosen when neither reward had a delay. A binomial test (two-tailed) was conducted on each bee's results, against a chance probability of .5. Two bees showed no significant preference for the sweeter reward even with no delay, and their results were excluded. Seven of 14 bees significantly preferred the sweeter delayed reward, 2 of 14 bees significantly

\section{Experiment 1}

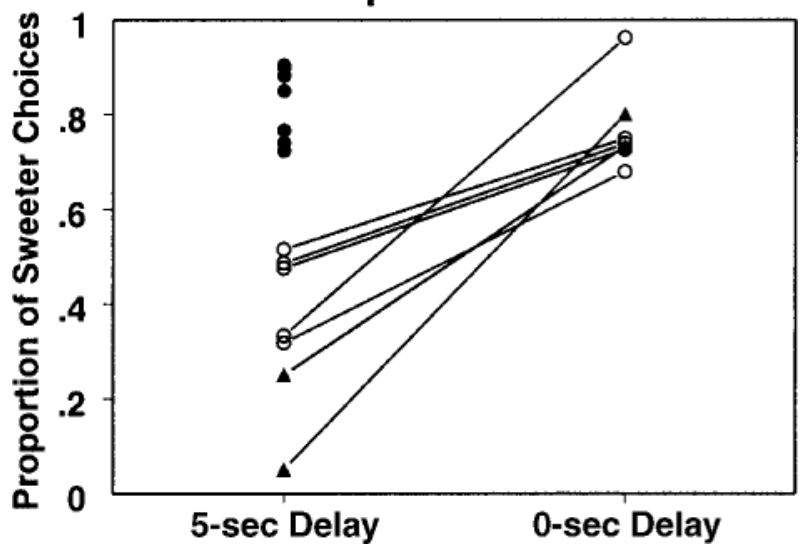

Figure 1. Proportion of choices of the sweeter reward by individual honeybees in Experiment 1. The left column indicates choices when the sweeter reward was delayed by 5 sec. Filled circles indicate bees that significantly preferred the sweeter reward, open circles indicate bees that were indifferent between the two rewards, and triangles indicate bees that significantly preferred the less sweet reward. The right column indicates the choices of those bees that did not significantly prefer the delayed sweeter reward when offered both rewards without delay. 
preferred the immediate less sweet reward, whereas 5 of 14 showed no significant preferences. Overall, $58.6 \%$ of the choices were for the sweeter delayed reward.

\section{EXPERIMENT 2}

Experiment 2 varied the size of the reward between the choices, rather than the quality of the reward. The smaller immediate reward was $10 \mu \mathrm{l}$, whereas the large delayed reward was inexhaustable. Pitting size against delay allowed comparisons with other species. Delay was manipulated differently. We thought that a hand reaching in to remove a glass might affect the behavior of bees. In this experiment, the rewards were inside a box, transparent at the top. The immediate reward was just inside the narrow entrance into the box, whereas the delayed reward was placed in the middle of the box, requiring a trip of a few seconds traveling into the box.

\section{Method}

\section{Subjects and Setup}

The subjects $(n=16)$ were recruited in the same way. Bees, individually trained and tested, were assigned to one of two groups: delay or no delay. The no-delay group, for whom the large reward was not delayed, provided a measure of choice accuracy in choosing the side with the large reward. The setup was modified from Experiment 1 . The landmark (cylindrical bottle) and blue and yellow poster paper were in place. The pieces of poster paper were covered with acetate so that they could be wiped with a damp cloth regularly. Two white plastic storage trays served as covers over the rewards (still in caps of $2.5-\mathrm{cm}$ diameter). The trays $(12.5 \mathrm{~cm}$ wide, $20 \mathrm{~cm}$ deep, $6.5 \mathrm{~cm}$ high, with a narrow protruding rim) were placed upside down on top of the blue and yellow poster papers, their front edges (facing the entrance from which the bees enter) lining up. The top of the upside-down trays had square holes that permitted light, but they were covered with acetate to prevent bees from entering or exiting. The sides and back of the trays were covered with white paper. At the front, a $2.5-\mathrm{cm}$ wide entrance was cut. On either side of the entrance was a piece of poster paper ( $5 \mathrm{~cm}$ wide), blue on the side near the cylindrical landmark, yellow on the other side. A gangplank of yellow poster paper $(2.5 \times 15 \mathrm{~cm})$ was placed at the entrance, protruding out by $2.2 \mathrm{~cm}$. It served to guide the bees to the rewards. The small reward (10 $\mu 1$ of 2:3 sugar solution in a cap) was just inside the entrance, whereas the large reward (a cap full of 2:3 sugar solution) was at the end of the gangplank for the delay group and at the entrance for the no-delay group. For half the bees, the large reward was on the right, whereas for the other half, it was on the left. Poster papers, trays, and gangplanks were regularly exchanged between sides, and sugar dishes were changed regularly.

\section{Procedure}

Delay group. A subject was first trained individually to find the sugar solution just inside the entrance of a cover, located centrally just in front of the landmark. In the training setup, the poster paper on both sides of the entrance was yellow. The gangplank was present, but not the blue and yellow square of poster paper. Once a bee could find a reward just inside the entrance, it was trained in three trials to find the reward farther and farther inside. On the third trial, the cap was at the back of the gangplank. Two forced-choice trials then took place. On each trial, the small reward alone was first set up. While the bee was consuming the small reward, the large $d e$ layed reward was set up. When the bee exited from the tray containing the small reward, the entire setup at that side was removed. Each bee then eventually found the large reward, drank to repletion, and was allowed to fly home. After the forced trials, both large and small rewards were set up, and the bee was allowed free choice for at least 37 trials (37-80). After the choice tests with the large delayed reward, the large reward was moved to just inside the entrance, and the bee was offered choices between large and small undelayed rewards (10-54 trials). (Two bees with $n<40$ showed clear results and were included.)

No-delay group. Each bee was trained individually until it could find the reward just inside the entrance of the box. Then two forced choices were provided in which the bee sampled both the small and the large rewards. Subsequently, testing continued over 2 days. The reason for testing over a 2-day period was to determine whether any improvement in performance could be detected on the 2 nd day. Because the delay group always had the no-delay tests after the delay tests, we wanted to see whether performance might improve on Day 2 of testing.

\section{Results and Discussion}

The honeybees again demonstrated a good deal of selfcontrol, producing results similar to those in Experiment 1 . The proportions of choices for the large reward made by individual animals in the two groups are shown in Figure 2. In the data shown in Figure 2, the first $n$ trials were not counted, with $n$ chosen to maximize the difference (in either direction) between Trials 1 to $n$ and Trials $n+1$ to the last trial. In all conditions, at least 20 trials from each bee were included. For experimental bees in the delay phase, we examined $n$ s ranging from 10 to 40 . For experimental bees in the no-delay phase, the range of $n$ s examined was 5-40. For the bees in the control group, the range of $n$ s examined was $10-40$. This was a formal and reasonable procedure for chopping out the first trials on which an animal might be expected to perform at chance. In the delay phase of the experimental group, 5 bees significantly preferred the large delayed reward by the binomial test, whereas 3 were indifferent between the large and the small

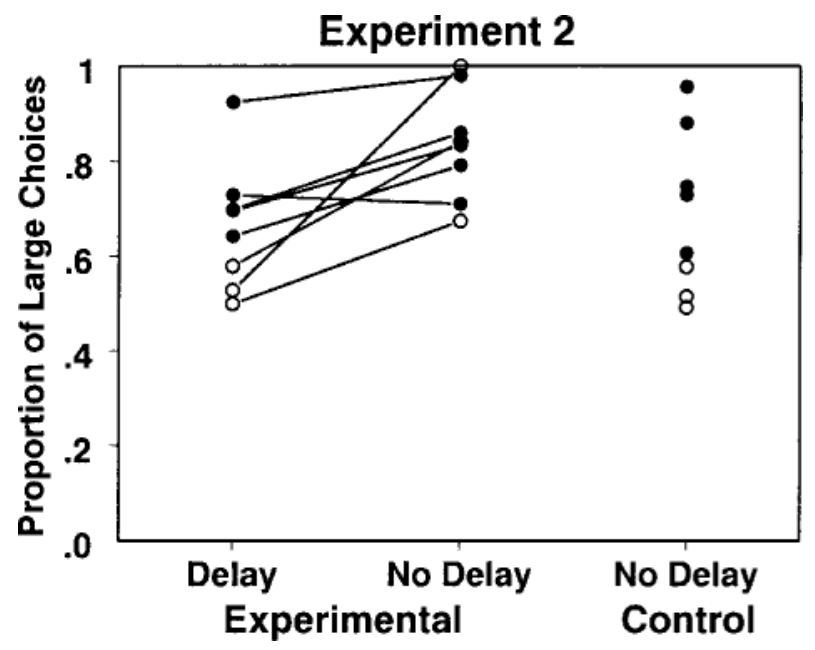

Figure 2. Proportion of large choices by individual bees in Experiment 2 . The experimental bees were first offered a choice between a delayed large reward and an immediate small reward and, in a second phase, a choice between a large and a small reward without delay. The control bees were offered a choice between large and small undelayed rewards throughout. 
rewards, and none preferred the impulsive option. The bees as a group preferred the large delayed reward $[t(7)=$ $3.413, p<.05$, two-tailed], with every animal over $50 \%$. In the second phase of testing, when the large reward also had no delay, all 8 bees chose the large reward significantly more often (Figure 2).

Figure 2 also shows the performance of control bees tested throughout with no delay for both rewards. Five of 8 bees chose the large reward significantly more often than the small reward, whereas 3 were indifferent. Performance was similar to that of the experimental animals and suggests that the task of discriminating the two choices was difficult for the insects. An analysis of variance (ANOVA) was undertaken to examine the extent of improvement on Day 2 for both groups of bees. We separated the data from the control group into 2 days. We treated the 2 days as two phases of testing, deleting the first $n$ trials of each day according to the rules used for the experimental bees. The ANOVA had group as a betweensubjects variable and phase as a within-subjects variable. The test found only a significant main effect of phase $[F(1,12)=4.82, p<.05]$. The interaction of group and phase was not significant $[F(1,12)=1.88]$. These statistics suggest that both groups of bees improved on Day 2 of testing, and to similar extents. This has two implications. First, the higher proportion of large choices under nodelay conditions in the experimental group may stem from improved discrimination of the two locations on the 2nd day of testing. Second, we may be underestimating the extent of self-control because the task of discriminating the two choices is difficult for the bees.

\section{EXPERIMENT 3}

In Experiment 3, we controlled the exact delay of the large reward, which was approximately three times the size of the small one. Parameters matched the study that Tobin and Logue (1994) chose as representative of rats, so that a direct comparison between bees and rats could be made.

\section{Method}

Between experiments, the beehive had moved to a new location. The experiment was run in a provisional laboratory in a small kitchen. The entrance system remained the same, and an arena consisting of a metal board was set up right at the entrance window, $2.5 \mathrm{~cm}$ below the entrance plank. The bases of two dark blue retort stands $(24 \times 14 \times 1.5 \mathrm{~cm}), 26 \mathrm{~cm}$ apart, equidistant from the entrance, and $10 \mathrm{~cm}$ from the wall below the entrance, held the rewards. The rewards sat on blue or green poster paper (12 cm square) covered by acetate $(14.5 \mathrm{~cm}$ square, regularly cleaned with a damp cloth), placed at the center edges of the retort stand bases. Just behind the poster papers, against the retort stand bases, stood cylindrical landmarks $(9 \times 9 \mathrm{~cm})$ of the same color. In between the blue and green cylinders stood a yellow cylinder of the same size. From the bee's view at the entrance plank, the green color was to the left, blue to the right. Blue and green were the target colors because pilot experiments showed that the bees could discriminate them and showed no preferences for one over the other. The reward caps were covered by transparent plastic $225-\mathrm{ml}$ cups (at the base) attached to strings, used to lift the cup with minimal disturbance to the bees. The strings were draped over attachments to the stem of the retort stands (at a 45-cm height).

Training procedures resembled those in Experiment 2. Each bee was first trained to find sugar water placed between the two targets. The cap sat on a rectangular piece of poster paper $(14 \times 7 \mathrm{~cm})$, half blue and half green. She was then given 2 training trials. A small reward $(17 \mu \mathrm{l})$ was set up on one retort stand. When the bee found that, a large reward (full cap) was set up on the other. No plastic cups were used for these 2 trials. For 2 bees, the large reward was on the green side, whereas for 3 , the large reward was on the blue side. Testing then began. The rewards were covered with plastic cups. The large reward had a delay of $6 \mathrm{sec}$, timed as in Experiment 1, whereas the small reward had a reward of $0.1 \mathrm{sec}$. A bee that chose the small reward always flew subsequently to the large reward and drank to repletion before flying home. Two bees were tested in a day, but from the training trials onward, only 1 at a time was allowed entrance to the laboratory. The bees were tested for most of a day (39-49 trials).

\section{Results}

For results, performance from Trial 6 of the test phase onward was counted. (The results were similar if the scoring methods of Experiment 2 were used.) One bee showed mostly impulsive choices (13.2\% for the large reward), whereas 4 showed mostly self-control $(68.2 \%, 90.0 \%$, $79.4 \%, 94.7 \%$ for the large reward). Four control bees were tested as well, with no plastic cups and, hence, no delay at either reward. One reward was sugar water, whereas the other was water. Again, not counting the first five tests, the bees chose the sugar water first $(100 \%, 73.3 \%, 97.6 \%$, $74.1 \%)$. The control and experimental animals did not perform differently $(F<1)$.

\section{GENERAL DISCUSSION}

In three laboratory experiments with free-flying bees, it was found that bees typically preferred a larger delayed reward over a smaller immediate reward. According to the metabolic hypothesis (Tobin \& Logue, 1994), the bees should show lower levels of self-control than do rats and pigeons, because they have faster metabolisms. The results from the bees in Experiments 2 and 3 can be compared with those of rats in two ways. First, self-control can be measured as deviations from the predictions according to the matching law, a method used by Tobin and Logue. We took $51 \mu 1$ to be the size of the large reward. In Experiment 2, we took the average of the median delays (time from entering the reward box to landing on the reward dish) of individual bees $(3.27 \mathrm{sec})$ to be the delay to the large reward and $0.1 \mathrm{sec}$ the delay to the small reward. By these calculations, the bees showed far more self-control than the rats (0.53 above matching law vs. 0.15$)$. Second, for Experiment 3, the matching law may be dispensed with, since the relevant parameters for bees and rats (in the experiment picked by Tobin \& Logue, 1994) matched. The bees again showed far more self-control than did the rats (69\% vs. $20 \%)$. We can conclude that honeybees show far more self-control than what is predicted by the metabolic hypothesis.

Tobin et al. (1996) found a high degree of self-control in two macaques. They proposed that relative brain size, 
as measured by Jerison's (1973) encephalization quotient, predicted self-control in a species. The encephalizationquotient was designed for vertebrates and cannot be applied to honeybees. But for the encephalization hypothesis to hold, bees must possess substantially larger relative brain size than do rats, a prospect we find unlikely.

A possible mechanism for self-control in foraging honeybees is precommitment. T. R. Zentall (personal communication, September 18, 2000) pointed out that for foraging bees, the effective time of reinforcement may not be at the gathering of nectar or pollen, but at the unloading of the forage back at the hive. This makes the effective delays of both the immediate and the delayed rewards in our experiments much longer. Under such conditions - that is, choosing well in advance of the smaller sooner reward-vertebrate animals tend to precommit to the larger delayed reward (e.g., Rachlin \& Green, 1972). This analysis predicts that any animal foraging "for the future" should display much self-control. Examples are gathering food for storage or to feed the young. This hypothesis is well worth testing.

Limitations to this study must be acknowledged. The comparisons are based on only two experiments with bees. We were inspired by Logue's $(1995,1998)$ ecological analysis to suggest that the honeybee should show much self-control - that is, because the forager is working mainly for the long-term benefit of her hive, the welfare of the hive being crucial for propagating her genes. This ecological hypothesis suffers from vagueness, making it lack quantitative precision. Unlike specific metabolic rate, the independent variable of the ecological hypothesis is not quantitative. A larger number of species also needs to be tested. Selecting a wide range of unrelated species, which might differ in many ways other than ecological variables predicted to affect self-control, might not be the optimal approach. Instead, it might be preferable to test a range of closely related animals (such as bees) that differ in the extent to which they work for the good of their nest, adopting the classic comparative method to study adaptation and cognition (Shettleworth, 1998). This approach might make it easier to quantify independent variables that predict self-control in a species.

\section{REFERENCES}

Ainslie, G. W. (1974). Impulse control in pigeons. Journal of the Experimental Analysis of Behavior, 21, 485-489.

Baumeister, R. F., Heatherton, T. F., \& Tice, D. M. (1994). Losing control: How and why people fail at self-regulation. San Diego: Academic Press.

BitTerman, M. E. (1996). Comparative analysis of learning in honeybees. Animal Learning \& Behavior, 24, 123-141.

Boeneme, R, Blakely, E. B., \& Poling, A. (1986). Runway length as a determinant of self-control in rats. Psychological Record, 36, 285288.

Chelonis, J. J., \& Logue, A. W. (1996). Effects of response type on pigeons' sensitivity to variation in reinforcer amount and reinforcer delay. Journal of the Experimental Analysis of Behavior, 66, 297-309.

Cheng, K. (2000). How honeybees find a place: Lessons from a simple mind. Animal Learning \& Behavior, 28, 1-15.

Collett, T.S., \& ZeIL, J. (1998). Places and landmarks: An arthropod perspective. In S. Healy (Ed.), Spatialrepresentation in animals (pp. 1853). Oxford: Oxford University Press.

Forzano, L. B., \& CoRry, R. J. (1998). Self-control and impulsiveness in adult human females: Effects of visual food cues. Learning \& Motivation, 29, 184-199.

Green, L., Fisher, E. B., Jr., Perlow, S., \& Sherman, L. (1981). Preference reversal and self-control: Choice as a function of reward amount and delay. Behavior Analysis Letters, 1, 43-51.

Green, L., Fry, A. F., \& Myerson, J. (1994). Discounting of delayed rewards: A life-span comparison. Psychological Science, 5, 33-36.

Green, L., Price, P. C., \& Hamburger, M. E. (1995). Prisoners' dilemma and the pigeon: Control by immediate consequences. Journal of the Experimental Analysis of Behavior, 64, 1-17.

Grosh, J., \& Neuringer, A. (1981). Self-control in pigeons under the Mischel paradigm. Journal of the Experimental Analysis of Behavior, 35, 3-21.

HePburn, H. R. (1986). Honeybees and wax: An experimental natural history. Berlin: Springer-Verlag.

JERISON, H. J. (1973). Evolution of the brain and intelligence. New York: Academic Press.

Logue, A. W. (1988). Research in self-control: An integrating framework. Behavioral \& Brain Sciences, 11, 665-709.

Logue, A. W. (1995). Self-control: Waiting until tomorrow for what you want today. Engelwood Cliffs, NJ: Prentice-Hall.

Logue, A. W. (1998). Self-control. In W. O. Donohue (Ed.), Learning and behavior therapy (pp. 252-273). Needham Heights, MA: Allyn \& Bacon.

Logue, A. W., Rodriguez, M. L., Peña-Correal, T. E., \& Mauro, B. C. (1984). Choice in a self-control paradigm: Quantification of experience-based differences. Journal of the Experimental Analysis of Behavior, 41, 53-67.

Mazur, J. E., \& Logue, A. W. (1978). Choice in a self-control paradigm: Effects of a fading procedure. Journal of the Experimental Analysis of Behavior, 30, 11-17.

RACHLIN, H., \& GREEN, L. (1972). Commitment, choice, and self-control. Journal of the Experimental Analysis of Behavior, 17, 15-22.

SeEley, T. D. (1985). Honeybee ecology: A study of adaptation in social life. Princeton, NJ: Princeton University Press.

SEELEY, T. D. (1995). The wisdom of the hive: The social physiology of honey bee colonies. Cambridge, MA: Harvard University Press.

Shettleworth, S. J. (1998). Cognition, evolution, and behavior. New York: Oxford University Press.

Tobin, H., Chelonis, J. J., \& Logue, A. W. (1993). Choice in self-control paradigms using rats. Psychological Record, 43, 441-453.

Tobin, H., \& Logue, A. W. (1994). Self-control across species. Journal of Comparative Psychology, 108, 126-133.

Tobin, H., Logue, A. W., Chelonis, J. J., Ackerman, K. T., \& MaY, J. G., III (1996). Self-control in the monkey Macaca facsicularis. Animal Learning \& Behavior, 24, 168-174.

(Manuscript received June 26, 2000; revision accepted for publication July 2, 2001.) 